\title{
A PRECISE AND RAPID METHOD OF MEASURING FRE- QUENCIES FROM 5 TO 200 CYCLES PER SECOND
}

\author{
By N. P. Case
}

\section{ABSTRACT}

After a brief discussion of some of the common methods of measuring frequencies from 0 to 200 cycles, a method is described which offers important advantages, combining a high degree of accuracy with ease and rapidity of use. The method depends on the fact that if a condenser be discharged through a resistance $n$ times per second (the condenser being charged to the same initial voltage each time), then the average voltage drop across the discharging resistance is directly proportional to $n$. The unknown frequency is made to control the number of times per second the condenser is discharged, and thus the voltage drop mentioned above is proportional to the unknown frequency. A circuit arrangement is described whereby this voltage drop is balanced through a sensitive galvanometer and high resistance, against a known fraction of the total voltage drop along a slide-wire resistance shunted around a storage cell. By first calibrating the system with an alternating current of known frequency, it is possible to read unknown frequencies directly off the slide wire.

A discussion of the sources of error and experimental determinations of the error actually observed, lead to the conclusion that, in the range from 5 to 200 cycles per second, the accuracy is always better than one-tenth cycle.

\section{CONTENTS}

I. Introduction -237

II. Early work

III. Final design

IV. Theory

V. Method of operation 241

VI. Accuracy obtainable

\section{INTRODUCTION}

During the course of the work carried on by the Bureau of Standards in the development of secondary standards of frequency, it is necessary to make daily measurements of the beat frequencies between various piezo oscillators used as secondary standards. As some of these secondary standard piezo oscillators do not vary in frequency from day to day by more than a few tenths of a cycle per second, the method of measuring the beat frequencies should be accurate to better than a tenth of a cycle per second. The frequency range of the beat notes to be measured is usually from 0 to about 100 cycles per second.

One method of measurement used is to record the beat frequency photographically on an oscillograph, at the same time impressing second signals on the record from a standard clock. As the constancy of the piezo oscillators improved, however, it became apparent that this method required too much time to be practicable. In order to obtain an accurate record by this means, it is necessary to let each 
exposure run for about 20 seconds. This gives a record which is very arduous to count, and the number of measurements to be made is such that the complete process of intercomparing all the piezo oscillators and reducing the results occupies too much time.

\section{EARLY WORK}

Consideration was given to the possibility of using a direct-reading frequency bridge, special consideration being given to the modified form of Hay's bridge, as described by Soucy and Bayly. ${ }^{1}$ This arrangement was not found to be entirely suitable for the purpose, however, for two reasons: (1) In order to secure adequate sensitivity in the region of very low frequencies used, it would be necessary to use an unreasonably large air-core inductance, and (2) as an aural balance would not be sufficiently accurate, because of the low sensitivity of the ear at these frequencies, it would be necessary to use a tuned vibration galvanometer, which is not considered practicable in view of the wide range over which it would have to be tuned.

The arrangement presented in this paper is an outgrowth of a system originally described by Maxwell ${ }^{2}$ for rough measurements of

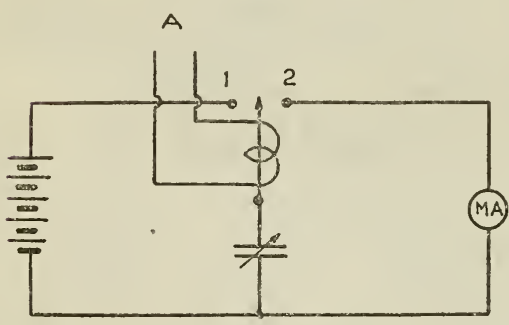

Figure 1.-Circuit arrangement for measuring frequency by means of deflection discharge of a condenser through a milliammeter capacity. Figure 1 is a circuit diagram of the arrangement as altered by Fleming and Clinton. ${ }^{3}$ The alternating current, the frequency of which is to be determined, is fed into a polarized relay at $A$. On one-half of each cycle the contactor is drawn to contact 1 , charging the condenser to the voltage of the battery. On the other half of each cycle, the contactor moves to contact 2, discharging the condenser through the milliammeter. Obviously, as long as the time constants of the circuits are kept low enough so that charge and discharge are complete within the limits of error of the measurement, the quantity of electricity which passes through the milliammeter in a given time interval depends solely on the number of times the condenser is discharged in that interval, and, hence, on the frequency of the alternating current applied to the polarized relay. Therefore, the reading of the milliammeter is directly proportional to the frequency. In practice, of course, the instrument has to be calibrated by applying a known frequency, say, 100 cycles per second, to the polarized relay, and then adjusting the battery voltage and the capacity until the meter reads 100 . Then if some other frequency is applied to the relay, the milliammeter reading will give the frequency directly. The accuracy, however, is only of the order of 1 per cont of the full-scale reading of the milliammeter, which, while sufficient for many laboratory purposes, was not great enough for the measurements required .

1 Soucy and Bayly, A Direct Reading Frequency Bridge for the Audio Range, Based on Hay's Bridge Circuit, Proc. 1. R. E., 17, p. 834; May, 1929.

${ }_{2}$ Maxwell, Electricity and Marnetism, sec. 775 , vol. II.

3 Fleming and Clinton, On the Measurement of Small Capacities and Inductances, Proc. Phys. Soc. London, 1.8, p. 386. See also F. $\Lambda$. Laws, Electrical Measurements, p. 373; 1917. 


\section{FINAL DESIGN}

It was recognized that the sensitivity of the above arrangement could be made amply sufficient by converting it to a null method and using a sentitive galvanometer. It was desirable also to have the readings directly in frequency. The circuit arrangement finally used is shown in Figure 2. The voltage divider $S$ is a slide-wire, with a thousand divisions on the scale, and accurate to the nearest quarter division at any point on the scale. The voltage is measured across the resistance $R$, through which the condenser $C$ discharges. The resistance $r$ is inserted to serve a double purpose; to keep the galvanometer from being greatly overdamped, since $R$ and $S$ are both of low resistance; and, more important, to limit the current that is drawn from the slide-wire. At first sight this appears to be an anomalous condition, since the adjustment is made to give zero reading on the galvanometer, but it must be remembered that a continuous voltage is being balanced against the average value of a pulsating voltage. This pulsating voltage is relatively very high during a very short part of the pulsation period, and is practically zero for most of the period. Therefore, there is only one instant during each pulsation when the galvanometer current is actually zero.

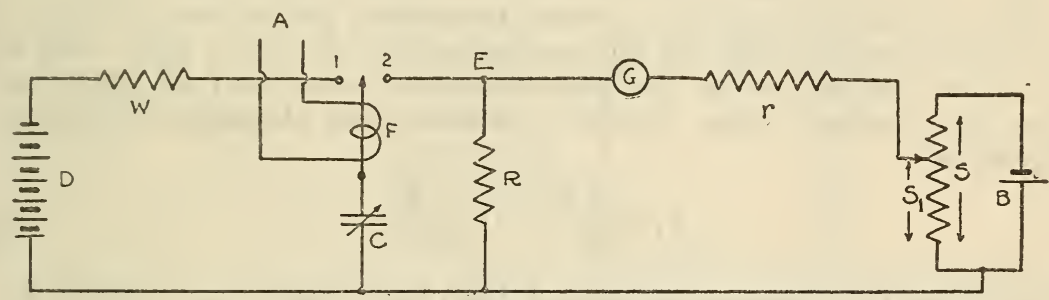

FIGURE 2.-Schematic diagram of circuit arrangement used for measuring low frequencies

The galvanometer current is limited to a small value, since the resistance $r$ (fig. 2) must be large for the settings of $S_{1}$ to be correct. In order that a small variation in $S_{1}$ may give an appreciable deflection, a high-sensitivity galvanometer must be used. As the accuracy of calibration of the slidewire is about $2 \frac{1}{2}$ parts in 10,000 , the resistance $r$ should be of such a value that the resistance of $S$ is not greater than 0.00025 of $r$. As $S$ is $7 \frac{1}{2} \mathrm{ohms}$, it follows that $r$ should be at least $30,000 \mathrm{ohms}$. A resistance of $50,000 \mathrm{ohms}$ was used, as that size happened to be available, and the galvanometer was of ample sensitivity. A damping shunt was affixed to the galvanometer and adjusted so that the instrument was not quite critically damped.

The resistance $R$ is low enough in comparison with $r$ so that the variations in the voltage of the point $E$, as the slider is moved from one end of $S$ to the other (the relay being open circuited), are negligible compared to the voltage of the battery $D$. If this condition is not fulfilled, the condenser will discharge to varying voltages, depending on the setting of $S$, and the reading of the latter will not be linear with the frequency of the discharge. A resistance of about $100 \mathrm{ohms}$ was used for $R$, and a capacicy of about $2 \mu$ f for $C$. Both are adjustable in small steps, although it would be sufficient if either one were so adjustable, in order to get a balance for the initial setting. The 
resistance $W$ is inserted to limit the initial charging current, and is not critical in value.

Values of circuit constants as used by the Bureau of Standards are as follows:

$$
\begin{array}{ll}
D=90 \text { volts } & r=50,000 \text { ohms. } \\
W=100 \text { ohms. } & S=7.5 \text { ohms. } \\
C=2.111 \mu \mathrm{f} . & B=2 \text { volts. } \\
R=100 \text { ohms. } &
\end{array}
$$

The galvanometer has a sensitivity of about 10,000 megohms, and a resistance of about $600 \mathrm{ohms}$.

\section{THEORY}

If the circuit conditions noted above, namely, $R$ and $S$ small compared to $r$, are complied with, the approximate theory becomes very simple. Referring to Figure 2 the moving contact of the relay $F$ is closed in position 1 and 2 , respectively, for sufficient time so that complete charge and discharge of condenser $C$ is obtained. The charge $q$ on $C$ is

$$
q=C e
$$

where $e$ is the voltage of battery $D$.

When $C$ discharges, the current takes two paths, one through $R$ and the other through the galvanometer $G$. If $r$ is so large that all the other resistances in the galvanometer circuit can be neglected, then the portion of the charge $q_{\theta}$ which flows through the galvanometer is

$$
q_{o}=q \frac{R}{R+r}=C e \frac{R}{R+r}
$$

If there are $f$ discharges per second, then the quantity per second is

$$
f q_{0}=f C e \frac{R}{R+r}
$$

The steady current through the galvanometer from the battery $B$, assuming $r$ large with respect to $S$, is

$$
i_{g}=\frac{S_{1}}{S} \frac{E}{R+r}
$$

where $E$ is the voltage of the battery $B$. The adjustment is made such that the effect of the current $i_{g}$ on the galvanometer is equal and opposite to the integrated effect of the pulsating current $f q_{g}$. Therefore, equating equations (3) and (4)

$$
\begin{gathered}
f C e \frac{R}{R+r}=\frac{S_{1}}{S} \frac{E}{R+r} \\
f=\frac{S_{1}}{S} \frac{E}{e C R}
\end{gathered}
$$

However, it is possible that the galvanometer may not integrate the pulses of current from the condenser properly, and, hence, may not read zero, even though equation (5) is satisfied. This condition is brought about by either of two causes: (1) The magnetic field at the 
galvanometer coil may not be constant, and (2) the coil itself may contain magnetic impurities. ${ }^{4}$

An experimental method used by Curtis and Moon to determine whether or not a given galvanometer integrates correctly is illustrated by the circuit of Figure 3. Care should be used with this arrangement in order to avoid damaging the galvanometer, especially if it is one of high sensitivity. The voltage of the battery should be small, the capacity of the condenser likewise small, and the resistances should be high enough to limit the initial currents on charge and discharge to safe values. If the galvanometer reads zero in the arrangement of Figure 3 , it may be safely assumed that it will integrate correctly in the frequency measuring network.

\section{METHOD OF OPERATION}

A tube-driven elinvar tuning fork was available for calibrating the slide-wire. The fork frequency was 99.98 cycles per second, remaining constant at all times to within 1 part in 10,000 .

The method of operation is extremely simple. The tuning fork output is applied at $A$ (fig. 2 ) and the slider on $S$ set so that the scale reading is $999_{8}$ (the last figure being beyond the calibration, must be estimated). Then the capacity of the condenser, the resistance $R$, or both, are varied until the galvanometer reads zero. If, now, the alternating current, the frequency of which is to be measured, is applied at $A$, the slider of $S$ is moved until the galvanometer again reads zero, all the other circuit constants remaining invariant. The frequency desired

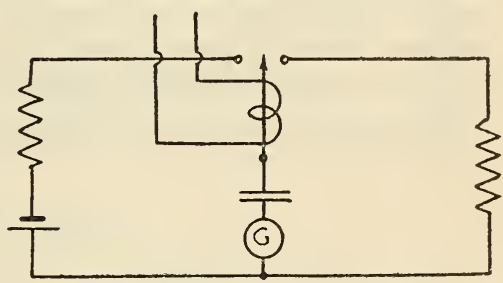

FIGURE 3.-Circuit arrangement used to determine suitability of galvanometer can then be read directly from the scale of the slide-wire. For instance, if the setting is $724_{9}$, the frequency is $72.4_{9}$ cycles per second.

If measurements of high precision are desired, it is advisable to redetermine the setting for the standard frequency before each series of measurements. This is necessary because of changes in battery voltages, and the changes in resistance and capacity of the circuit elements due to temperature changes. The battery circuits should be closed for a sufficient length of time before measurements are made so that the batteries and resistances can come to a condition of equilibrium.

For frequencies below five cycles per second the individual impulses to the galvanometer become objectionable and the balance point can not be accurately located. However, such low frequencies can be determined readily by merely counting the swings of the needle of the milliammeter (which is in the plate circuit of the last tube of the beat note amplifier) over a short period of time. A stop watch can be conveniently used.

In order to extend the range to higher frequencies it is only necessary to set the point for calibration at some lower point of the slide-wire and multiply the scale accordingly. For example, under the condi- 
tions given above, if instead of using 99.98 for the first setting, 49.99 is used, then 99.98 will represent 200 cycles per second. The limitation is, of course, in the frequency to which the relay will respond. The limitation of the relay used is about 200 cycles per second.

\section{ACCURACY OBTAINABLE}

Although the slide-wire is only claimed to be accurate to a quarter of a division, corresponding to 0.025 cycle per second, settings on a given frequency will usually repeat to 0.01 cycle per second.

The linearity of the scale has bean verified in two distinct ways. (1) A constant-speed alternator with 100-cycle and 10-cycle rotors on the same shaft, assuring that the one frequency is exactly one-tenth of the other, was available. The slide was balanced at 100 cycles per second, and then the 10-cycle frequency was measured in the regular manner, the scale reading obtained was $10.02 \pm 0.005$ (estimated) cycles per second. This error is less than the error of calibration of the slide-wire. (2) Numerous checks were also made at various odd frequencies, reading the frequency on the slide, and recording it on the oscillograph at the same time. These checks consistently agree within 0.03 cycle per second. Hence the maximum error is well within 0.1 cycle per second. No checks were made above 100 cycles, since all measurements desired were below this value.

Washington, March 8, 1930. 